Horizontes. Revista de Investigación en Ciencias de la Educación

ISSN 2616-7964

octubre-diciembre, 2017

Volumen 1, Número 4

pp. 37-45

www.revistahorizontes.org

\title{
Educar para la paz en el ámbito de la escuela: Lineamientos para una pedagogía humanista
}

\author{
Educating for peace in the school setting: Guidelines for a \\ humanistic pedagogy
}

\section{José Humberto Figueroa} jhfiap@gmail.com

Instituto Universitario de la Región de los Llanos

Recibido: 15-04-2017 / Revisado: 20-04-2017 / Aceptado: 20-05-2017 / Publicado: 09-10-2017

\section{RESUMEN}

El presente artículo se plantea el análisis de la situación actual de la escuela en relación al enfoque pedagógico asumido que no ha contribuido a dar vida a una pedagogía humanista que coadyuve a educar para la paz, se parte de un diagnostico apoyado en la revisión documental, planteando a nivel de propuesta: lineamientos de una nueva educación humanista, cuyo norte sea contribuir con la Paz. En el nivel metodológico se inicia con una revisión documental y se analiza el marco normativo en Venezuela, obteniendo así un diagnostico que lleve a la propuesta de un proyecto factible lineamientos para una pedagogía humanista.

Palabras clave: Educación para la Paz, Pedagogía Humanista, Enfoques pedagógicos, Educación en Derechos Humanos
ABSTRACT

This article presents the analysis of the current situation of schools in relation to the pedagogical approach assumed that has not contributed to give life to a humanistic pedagogy that helps to educate for peace, is based on a diagnosis supported by the documentary review, proposing at the proposal level: guidelines for a new humanist education, whose goal is to contribute to Peace. At the methodological level begins with a documentary review and analyzes the normative framework in Venezuela, obtaining a diagnosis that leads to the proposal of a feasible project guidelines for a humanistic pedagogy.

Key words: Education for Peace, Humanistic Pedagogy, Pedagogical Approaches, Human Rights Education 
INTRODUCCIÓN

La educación en Derechos Humanos constituye en el presente un gran reto de cara a las demandas sociales que la sociedad presenta en sus exigencias de dar respuestas a las tensiones socializante la pérdida de cohesión que se manifiesta en el deterioro de la identidad colectiva e individual y la tensión existente entre un individuo que va perdiendo los referentes morales, en especial de los valores de convivencia, llevando eso a la presencia de situaciones donde la violencia crece. Esta situación arropa a un número enorme de sociedades en el nivel global, en el que Venezuela no es ajena, siendo un proceso complejo que tiene raíces estructurales, donde la cultura y el modelo de civilización occidental han entrado en una fase de crisis, no obstante reconociendo que en otrora esos componentes que dieron vida a esta civilización, permitieron la emergencia de los derechos humanos en el siglo XX con la Declaración Universal de 1948, sin embargo se observa con preocupación que hoy pareciera no existir un norte humanista que permita guiar a la sociedad en la búsqueda del bien común, de manera que los anti-valores van protagonizando los cambios y el afán de controlar las riquezas materiales bajo un accionar individualista marcado por un egoísmo social, a tal punto que ese conjunto de relaciones sociales entran en tensión y son las que dan vida a la sociedad muestran signos de deterioro, siendo la violencia una expresión anómica de esos cambios donde las sociedades no logran sostener una cohesión positiva.

En ese contexto, entender ¿qué ha pasado con la sociedad?, plantea desde el presente estudio, en un nivel de propuesta, un diagnóstico de la situación general de las escuelas en relación al enfoque pedagógico de manera de proponer una pedagogía humanista que permita educar para la Paz, propuesta que se nutre desde la revisión documental y se alimenta ante los acelerados cambios que muestran no pocas tensiones sociales, donde esa misma escuela entró en una etapa de cuestionamiento porque su papel formador debe ser repensado, aun reconociendo que hay propuestas de intervención desde las escuelas, estos han sido poco efectivos en su efecto de educar para la vida y la paz, en especial en los niveles de educación primaria que son claves en la internalización de los valores que definirán la vida adulta de ese niño.

La propuesta Hacia una Pedagogía Humanista en la Escuela pretende aportar elementos para una praxis pedagógica más vivencial que conecte el espacio del aula y de la escuela con las familias, proceso que debe iniciar desde la reflexión involucrando a los maestros que hacen vida en primaria, los directivos, de manera de identificar aquellos elementos de la cultura escolar que frenan la construcción de una cultura sustentada en valores para la paz, por tanto la gestión y praxis pedagógica son importantes porque devienen en ejemplos.

Se trata de ir ganando espacios desde los cuales se generen cambios que lleven a una formación en valores para la Paz, en especial, niños y adolescentes que viven en un contexto de violencia y que reproducen la violencia también.

\section{CAMINO METODOLÓGICO}

Para dar cumplimiento a la estructura del trabajo, des el punto de vista metodológico se asume la revisión documental para tomar los elementos del contexto en relación al problema de la violencia escolar y se plantea de acuerdo al tipo de investigación, siguiendo al Manual de la UPEL, de un Proyecto Factible, que: "consiste en la investigación, elaboración y desarrollo de una propuesta de un modelo operativo viable para solucionar problemas, requerimientos o necesidades de organizaciones o grupos sociales; puede referirse a la formulación de políticas, programas, tecnologías, métodos o procesos", 
(p. 13), que inicia desde el diagnóstico y descripción del contexto general social donde está inserta la escuela, para llegar a la propuesta de Taller: Pedagogía Humanista hacia una educación para la paz dirigido a maestros y directivos y finalmente, presentar unas reflexiones teóricas que presenten los principales nudos críticos que frenan la construcción de una cultura de paz desde las escuelas.

\section{DISCUSIÓN Y RESULTADOS}

Crisis de la sociedad y deterioro de la función formativa de la escuela.

El mundo de hoy globalizado si bien muestra profundos cambios que apuntan a la modernización de la sociedad, cambios en el nivel de vida material y tecnológico que han llevado a una mayor calidad de vida y bienestar, simultáneamente también se van identificando procesos de conflictividad porque esos cambios se han construido desde relaciones de poder que en lo económico y social son muy desiguales, en este caso, la desigualdad social ha sido una condición negativa que muestra que el progreso ha descuidado al mismo ser humano, donde las prioridades pasan a ser el afán de acumular riquezas sin importar realidades concretas de miseria, conflictos y dolor que definen la vida cotidiana de muchos colectivos e individuos en todo el planeta, donde el principal problema pasa a ser la pobreza, que de acuerdo al Banco Mundial la misma cambia de acuerdo al nivel de ingresos estando entre ellas: pobreza extrema y hambruna, que hacen referencia a las personas cuyos ingresos no superan un dólar diario, siendo esto una condición que en definitiva potencia la pobreza y todos los problemas derivados de ella donde la violencia va creciendo, lo que coadyuva a reproducir situaciones anómicas que muestran como el equilibrio social se tensiona en individuos y grupos marginados de la sociedad que no han sido incorporados al desarrollo.

La pobreza material y social es un rasgo del presente que ha sido analizado por los organismos mundiales y ha sido reconocida como tal en los objetivos del milenio y en la agenda post-desarrollo del 2015 de la ONU, donde se reconoce que debe darse vida a acciones más activas que lleven a procesos reales de inclusión, una inclusión efectiva, en virtud que esa desigualdad social sigue creciendo y ha llevado a la sociedad a la perdida a un proceso sostenido de deterioro de los valores humanos en un presente cuyo norte humano de desdibuja, se confunde cuando observas una realidad muy caótica y llena de profundas narrativas de miseria y dolor humano. La violencia también forma parte de esos relatos del presente y ha estado presente a lo largo de la evolución de la sociedad moderna. Sin embargo, la sociedad ha venido cambiando con un mayor nivel de madurez, al punto que en el siglo XX la perspectiva de los Derechos Humanos adquirió universalización con la materialización de la Declaración Universal en 1948, lo que devino en una clara conciencia moral y jurídica de la humanidad ante lo que representó los horrores de la guerra. En ese sentido, desde el mismo preámbulo se establece:

Considerando esencial que los derechos Considerando que la libertad, la justicia y la paz en el mundo tienen por base el reconocimiento de la dignidad intrínseca y de los derechos iguales e inalienables de todos los miembros de la familia humana; Considerando que el desconocimiento y el menosprecio de los derechos humanos han originado actos de barbarie ultrajantes para la conciencia de la humanidad, y que se ha proclamado, como la aspiración más elevada del hombre, el advenimiento de un mundo en que los seres humanos, liberados del temor y de la miseria, disfruten de la libertad de 
palabra y de la libertad de creencias; humanos sean protegidos por un régimen de Derecho, a fin de que el hombre no se vea compelido al supremo recurso de la rebelión contra la tiranía y la opresión;

Resaltando en los enunciados de la Declaración Universal el valor de la dignidad de la persona por encima de todo, donde la igualdad y la libertad pasan a ser fundamentales para construir un mundo humano, donde se respeta la singularidad del individuo que reconoce y es reconocido por los otros, además, donde la paz y la justicia pasan a ser valores claves para la convivencia social. Este salto cualitativo, sin duda marcó un punto de inflexión hacia la protección de la dignidad humana, que con los derechos humanos consagrados en la Declaración permitió definir un conjunto de normas al alcance de las personas, de los grupos y colectivos para exigir el respeto de sus derechos, de la misma manera también especificados con los dos Pactos internacionales, donde se definen los derechos en general y las condiciones que establecen responsabilidades y límites a la acción de los Estados, como lo es el caso del Pacto Internacional de Derechos Civiles donde se establecen normas vinculantes.

En el ámbito nacional, nuestro país se integra a la normativa internacional de los derechos humanos, incluso con rango constitucional como así lo establece la Constitución Nacional en su Artículo 23 :

Los tratados, pactos y convenciones relativos a derechos humanos, suscritos y ratificados por Venezuela, tienen jerarquía constitucional y prevalecen en el orden interno, en la medida en que contengan normas sobre su goce y ejercicio más favorables a las establecidas por esta Constitución y en las leyes de la República, y son de aplicación inmediata y directa por los tribunales y demás órganos del Poder Público.
De esta manera, el marco legal venezolano es una fortaleza para el logro de los derechos humanos, no obstante, la experiencia vista desde la praxis muestra un proceso de deterioro en lo social, donde las estadísticas reflejan una economía en crisis y un proceso marcado por la conflictividad, donde la violencia ha crecido exponencialmente, ubicando a nuestro país como el segundo con mayor tasa de homicidios en el mundo; "Venezuela fue ubicada como el segundo país más violento del mundo, al tener una tasa de homicidios de 53,7 por cada 100.000 habitantes, según un informe elaborado por la Oficina de la Organización de las Naciones Unidas contra la Droga y el Delito".

Esta situación de violencia sin duda tiene raíces estructurales, donde el enfoque del desarrollo aplicado en el país ha dado vida a una cultura del facilismo, donde el Estado es visto como aquel proveedor, lo que no ha permitido desde la dinámica de un modelo rentista, potenciar una cultura del trabajo y del esfuerzo colectivo, al punto que hay que reconocer que el agotamiento del modelo rentista ha puesto de manifiesto, la crisis de un modelo societal donde si bien hay definiciones generales desde actores no gubernamentales importantes, ellas no han tenido la incidencia en las políticas concretas implementadas desde el poder en el marco de la revolución bolivariana, de manera de coadyuvar en la transformación de la sociedad, donde la política educativa en todos los niveles, si bien en su diseño teórico plantea un cambio de paradigma orientado a dar vida a una pedagogía humanista que prioriza el convivir y el ser, ello resulta inefectivo cuando es rastreado en la realidad escolar, más en los ambientes de la escuela venezolana, que presenta estadísticas que evidencian un aumento de la violencia escolar y que llevan a preguntarse: ¿por qué teniendo la Escuela un currículo donde se integra el eje transversal los valores y la educación en 
Derechos Humanos persiste una violencia entre pares que muestra una crisis en valores, en especial el respeto y la tolerancia?. Lo que debe llevar a la reflexión pues la escuela representa una institución que por antonomasia es clave en la formación en valores, modeladora en la conducta de los niños, además su función formadora es reconocida como la tal en la Constitución y aparece reflejada así en la Ley Orgánica de Educación que establece en su artículo 3:

la formación en una cultura para la paz, la justicia social, el respeto a los derechos humanos, la práctica de la equidad y la inclusión...Se consideran como valores fundamentales: el respeto a la vida, el amor y la fraternidad, la convivencia armónica en el marco de la solidaridad, la corresponsabilidad, la cooperación, la tolerancia y la valoración del bien común, la valoración social y ética del trabajo, el respeto a la diversidad propia de los diferentes grupos humanos.

De esa manera, vista la importancia que tienen los valores en su inserción en el currículo y de cómo la transversalidad resulta un imperativo al cual hay que darle vida en el contexto escolar, como bien lo señala (Magendzo, s/f) cuando plantea la existencia de una crisis de situación y en donde:

La coexistencia de estas incertidumbres y de estas certezas configura un cuadro de diversidad cultural que, a juicio de algunos, es similar a una crisis moral. Sin lugar a dudas estamos enfrentados a una búsqueda por nuevos paradigmas al interior de la sociedad en su conjunto. El paradigma que está hoy en vías de cambio -y que privilegia una concepción racionalista del ser humano- ha dominado nuestra cultura y nuestra convivencia social por siglos .

El cambio de paradigma donde ahora la transversalidad sustentada en una Educación en Valores y en Derechos Humanos, reconoce la importancia del conocimiento y del hacer en cuanto a que son importantes en la formación académica de los educandos, pero también desde la teoría valora el convivir y el ser, siendo estos medulares en la formación del nuevo hombre, acciones que en al ámbito práctico aportarían mucho para la transformación en un en el contexto crisis societal en el país, de allí la importancia de la propuesta de una Pedagogía Humanista en el Contexto de la Escuela, que de vida a cambios reales en las relaciones que definen la enseñanza - aprendizaje que en este momento la sociedad demanda, dar vida a procesos de pedagógicos que se construyan en la búsqueda de la excelencia académica, pero que simultáneamente incorporen lo humano en el centro de la enseñanza. El convivir y el ser no pueden estar ajenos en la formación educativa, por tanto, la Pedagogía Humanista planteada en esta propuesta tiene como intención contribuir con la formación de estudiantes que teniendo un amplio conocimiento de las realidades también este formado para integrarse en la sociedad en un clima de respeto y tolerancia con los otros, una convivencia social sana. No es esto una tarea fácil, requiere de la mayor suma de voluntades, que en el caso planteado a lo interno de las escuelas debe partir del reconocimiento de la necesidad de repensar la escuela en un contexto donde se integren todos los actores: Escuelas, Familias y Sociedad. Una tríada clave para dar vida a la transformación.

\section{Hacia una pedagogía humanista en el contexto de la escuela.}

Los procesos de transformación que muestran una sociedad en constante cambio abarcan también al conjunto de relaciones culturales, donde la escuela es repensada de cara a construir un mundo más humano, en especial cuando se reconoce que hay una tensión entre la identidad individual y social, donde las personas no logran cohesionarse a partir de valores humanos que en otrora 
permitieron definir la modernidad. El progreso material se ha estructurado de manera vertiginosa, pero éste ha puesto en tensión el sentido ético-moral de la sociedad y de los individuos, en especial cuando se trata de educar para la paz, en una convivencia donde la persona se sienta respetada y reconocida en los otros, en mundo donde la convivencia social está en crisis. De allí que la propuesta de una pedagogía humanista den el contexto de la escuela cada día adquiere relevancia y aun cuando es reconocida en el mundo de las reformas y pensada por grandes humanistas como Paulo Freire en su texto Pedagogía del Oprimido, su concreción efectiva dista mucho de alcanzarse porque aún siguen estando presentes ciertos elementos de orden estructural que devienen en frenos para la transformación, que deben ser reflexionados a lo interno de las escuelas a través de la incorporación de actividades que sean generadoras de diagnósticos que sirvan d insumos para la planificación e instrumentación de los contenidos académicos y transversales del currículo. En este sentido, la educación orientada a generar una cultura de paz y de los derechos humanos se conecta con la misma realidad escolar y involucra también la integración de las familias, siendo éste un aspecto clave para dar vida a esa propuesta. Reconociendo eso se enuncian los objetivos.

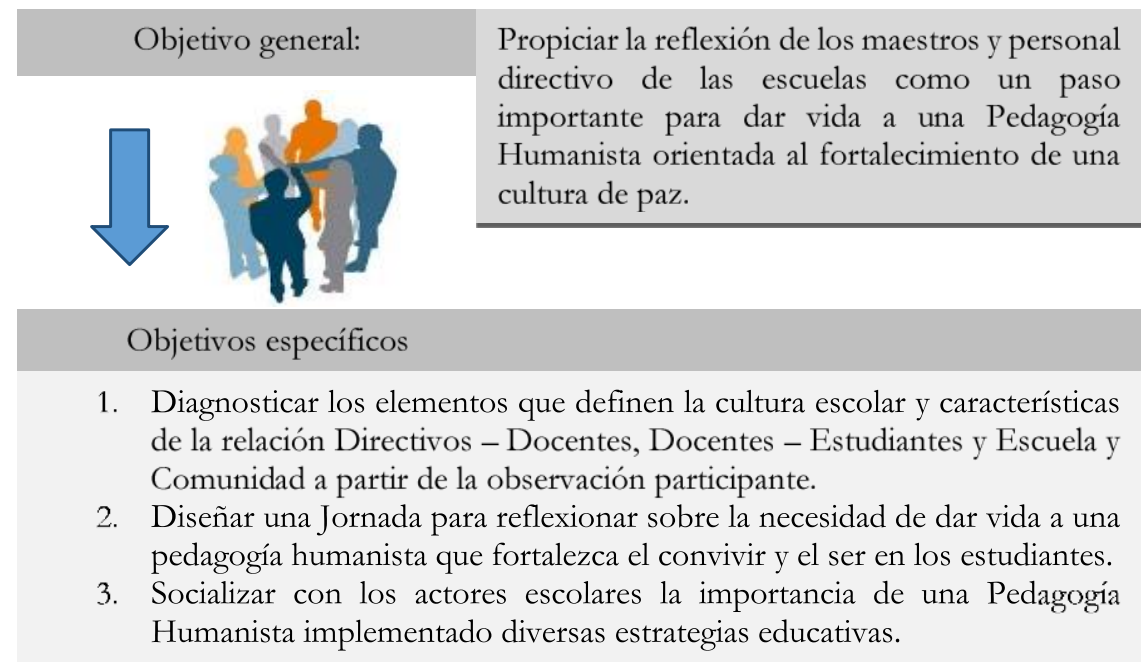

Siendo la propuesta la de generar espacios para la reflexión que permitan a los actores que hacen vida en la escuela identificar los principales problemas que desde el enfoque pedagógico tradicional se presentan como frenos para una construir una cultura de paz en la escuela, siendo ello corroborado cuando se reproducen situaciones de violencia donde el maestro puede incluso puede también ser responsable porque no logra dar el salto cualitativo en la manera enseñar, limitándose a reproducir en los estudiantes contenidos formales - teóricos que pueden ser en el conocer, pero no articulado con el convivir y el ser. Por tanto, pensar en dar vida en jornadas de reflexión es un paso necesario hacia la pedagogía humanista, lograr que los actores escolares identifiquen sus problemas y que la violencia como tal es una situación que tiene que ver tanto con la sociedad como la misma escuela, ahora, llegado a ese punto de reconocimiento sin duda será este un gran paso para orientar fortalecer el convivir y el ser en la escuela, lo que sólo se lograra con relaciones dialogantes donde la comunicación sea transversal en todos los ejes y que desde el punto de vista de la cultura escolar permita crear un contexto 
coherente con la propuestas, en donde las distintas relaciones se construyan con el ejemplo.

Los Momentos que abarcan las distintas etapas de las Jornadas de Reflexión para dar vida a una Pedagogía Humanista son los siguientes:

1. Momento Inicial asumido por el investigador: orientado a identificar desde la observación participante los problemas que frenan la instrumentación de una pedagogía humanista para el convivir y ser en los estudiantes. Este momento se complementa con el diagnóstico en el contexto en el que se desarrolle el taller con lluvia de ideas, de manera de registrar los problemas planteados por los sujetos involucrados, léase: maestros y directivos.

2. Momento de Desarrollo: en esta etapa se involucra el uso de una estrategia pensada para la sensibilización de manera de recrear los límites de la pedagogía tradicional en el tipo de relaciones verticales que reproduce el maestro con sus alumnos y la dirección con los maestros. La Dramatización puede en este sentido, ser una pertinente estrategia para lograr el objetivo.

3. Momento de Cierre: valorando los aportes de los participantes en el desarrollo del taller y colocando el énfasis en los aspectos que puedan fortalecer la práctica de una pedagogía humanista en la escuela. Esto lleva a sugerir hacer un seguimiento desde el aula, lo que debe llevar a las escuelas a tener personal capacitado para monitorear la progresividad del cambio. No existirá retroalimentación en cuanto al sentido del taller sino se instrumentan en las siguientes situaciones: aula de clases donde se dan las relaciones maestras estudiantes, relaciones de comunicación entre directivo y maestros e relaciones entre escuela $\mathrm{y}$ familia visto desde aquellas instancias $y$ espacios de encuentro que la escuela puede usar para alcanzar ese objetivos.

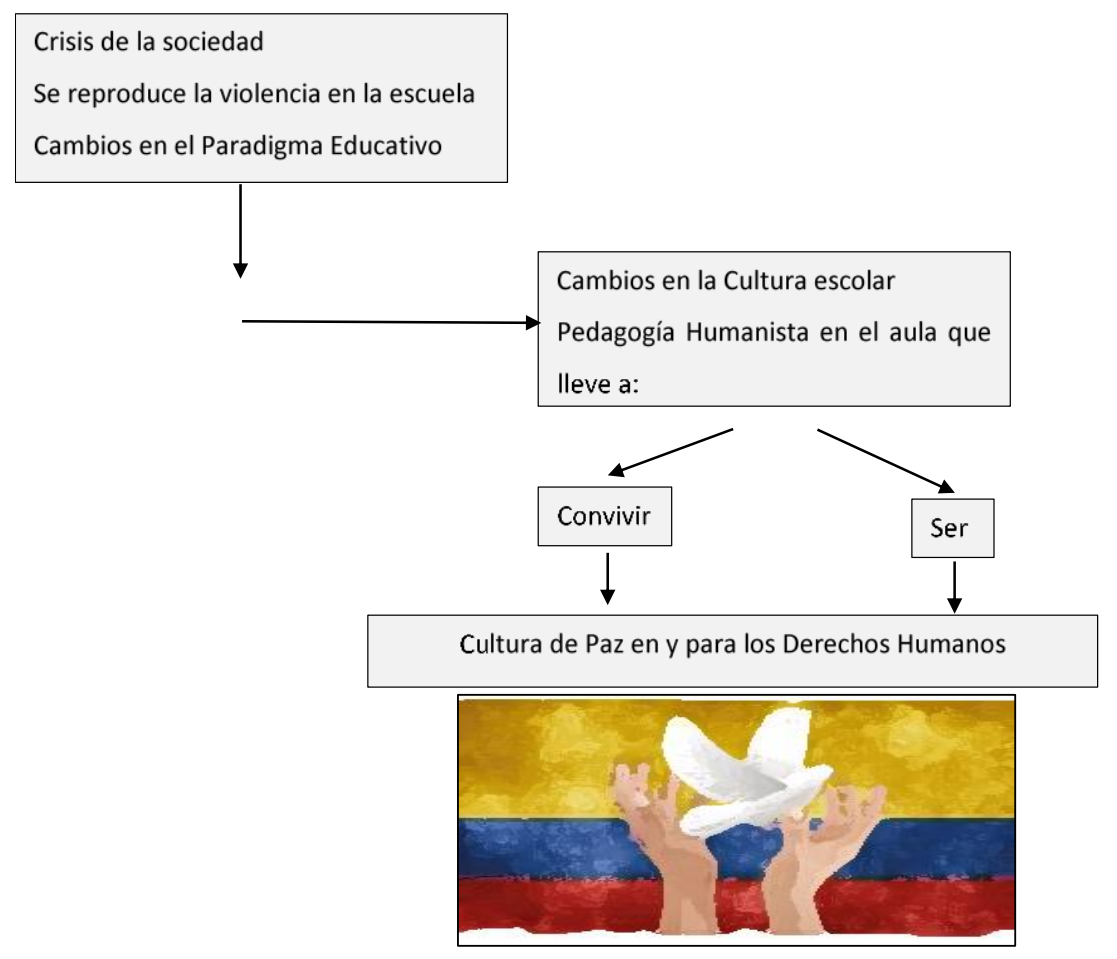

Horizontes. Revista de Investigación en Ciencias de la Educación. Vol. 1, Nro. 4, Octubre-Diciembre, 2017 
A MANERA DE REFLEXIÓN

Educar en derechos humanos, en especial en una educación orientada a fomentar una cultura para la paz en el presente representa un gran reto, que en el nivel de la actual crisis de país exige la mayor suma de voluntades en un contexto de transformaciones profundan en lo moral y en las estructuras materiales donde, en ambas, se presentan muchos déficits, que en general han llevado al deterior de la cohesión social, donde los mecanismos de integración asumidos por las familias y escuelas como instancias de socialización presentan quiebre, al nivel que la educación en valor reduce su significación en un contexto donde la violencia escolar entre pares es expresión también de la violencia más amplia de la sociedad venezolana, ante esos cambios negativos la identidad de la persona, en especial la de los niños y adolescentes que van formándose entran en tensión con una sociedad que viene perdiendo el norte humano.

La crisis de los valores, no sólo expresa la crisis social en cuanto a la perdida de la cohesión social en ambientes donde las normas morales y el horizonte ético dan paso a nuevas prácticas culturales y nuevas relaciones sociales que muestran conductas sociales impregnadas por la violencia social, lo que afecta todos los ámbitos de la vida socioinstitucional, donde la Escuela y las familias deben transformarse para dar respuestas a las necesidades de formar en valores en y para los Derechos Humanos.

La Educación en Derechos Humanos debe ser una educación que trascienda lo meramente teórico para dar paso a la definición de componentes que desde la acción pedagógica den vida a la dialéctica entre el ser individual y social, de manera que la población escolar niños y adolescentes asuman desde la vivencia el significado de la educación para la paz, se reconoce así que ésta sólo se internaliza desde lo testimonial, donde el mundo afectivo-emocional resulta medular en ese proceso de formar en valores. Esto significa que la escuela para dar vida a estrategias eficaces, más allá de un aprendizaje teórico, que trascienda al ámbito de lo humano vivencial, desde una educación con ejemplos y en eso el maestro es una pieza importante porque representa una figura modeladora al igual que los padres, de allí que la propuesta exige revisar los aspectos propios de la cultura escolar, modificando ese viejo modelo autoritario pedagógico, de manera que la propuesta de una cultura educativa que potencie y de vida a la educación en y para los Derechos Humanos para la paz tenga viabilidad en un contexto escolar más flexible desde lo pedagógico. Esa observación se plantea porque hay evidencias que muestran como la cultura escolar sigue presentando elementos de resistencia al cambio tanto en la gestión como en lo testimonial de ese de valores que no logra concretarse desde los ejemplos, hay experiencias muy importantes, pero también persiste los problemas vinculadores con la cultura escolar, más cuando se observa un repunte de la violencia escolar que ha llegado a niveles que deben llamar la atención.

Es por ello que la propuesta de desarrollar talleres para la reflexión resulta importante para generar procesos de sensibilización en los actores que hacen vida dentro de las escuelas, de manera que puedan ellos dar vida a un enfoque pedagógico humanista que valorice los pilares del convivir y del ser. Una educación en y para los derechos humanos resulta medular para construir una cultura de paz, donde la tolerancia $y$ el respeto deben ser ejemplificados en el conjunto de relaciones que dan vida a la escuela. Por tanto, el enfoque humanista se tiene que recrear desde lo testimonial, con ejemplos, donde los maestros trasciendan lo teórico-formal y den vida a una pedagogía que tome en cuenta el convivir y el ser, esto es, un ser humano que siendo singular se relaciona con otros, un ser humano 
afectivo, un ser humano al cual hay que formar en valores. Con el testimonio y con los ejemplos se lograrán muchos cambios.

El reto de formar en valores es enorme, es una tarea compleja porque involucra una serie de cambios y es ahí que la escuela está llamada a dar vida a esa transformación. Generar espacios para la reflexión en ese sentido es un paso inicial importante

\section{REFERENCIAS}

Banco Mundial (s/f). Pobreza Extrema y Hambruna. Referencia en línea: http://www.bancomundial.org/temas/o $\mathrm{md} /$ definiciones.htm

Constitución de la República Bolivariana de Venezuela. (1999). Caracas, Venezuela: Ediciones de la Presidencia de la República.
Declaración Universal de los Derechos Humanos.(s/f). Recuperado en http://www.un.org/es/documents/udhr /

Diario El Nacional. Recuperado en: http://www.elnacional.com/sociedad/Ve nezuela-segundo-pais-violentomundo_0_641336191.html. Consultado: 24/02/2016.

Freire, Paulo (s/f): Pedagogía del oprimido. Recuperado en: http://www.bvsst.org.ve/cumentos/pnf/ pedagogia_del_oprimido.pdf. Consultad: 18/02/2016.

Magendzo, Abraham (s/f): Los Derechos Humanos. Un objetivo transversal del currículum. Recuperado en: http://dip.una.edu.ve/ddhh/educaciondd $\mathrm{hh} /$ paginas/2- 